DE'88 003283

\title{
HEAVY ION EXCITATION OF GIANT RESONANCES - THE NEXT GENERATION
}

F. E. BERTRAND, J. R. BEENE, D. J. HOREN

Oak Ridge National Laboratory

Oak Ridge, Tennessee 37831 , U.S.A.

\author{
Invited Paper Presented at the \\ First. Topical Meeting on \\ Giant Resonance Excitation in Heavy-Ion Collisions \\ Legnaro, Italy' \\ September $21-25,1987$
}

DISCLAIMER

\begin{abstract}
This report was prepared as an account of work sponsored by an agency of the Unitei States Government. Neither the United States Government nor any agency thereof, nor any of their employees, makes any warranty, express or implied, or assumes any legal liability or responsibilit: 'or the accuracy, completeness, or usefulness of any information, apparatus, product, or prociss disclosed, or represents that its use would not infringe privately owned rights. Reference herein to any specific commercial product, process, or service by trade name, trademark. manufacturer, or otherwise does not necessarily constitute or imply its endorsement, recom. mendation, or favoring by the United States Government or any agency thereof. The views and opinions of authors expressed herein do not necessarily state or reflect those of the United States Government or any agency the-eof.
\end{abstract}




\section{HEAYY ION EXCITATION OF GIANT RESONANCES - THE NEXT GENERATION}

F. E. BERTRAND, J. R. BEENE, D. J. HOREN

Oak Ridge Nationa1 Laboratory,* Oak.R.idge, Tennessee 37831, U.S.A.

figures

The prospects for high energy heavy ion excitation of giant resonances are discussed. A brief review is provided of the results obtained to date using low/medium energy heavy ions and it is suggested that those results show no substantial advantage of heavy ions for giant resonance excitation.

Calculations are presented that show that coulomb excitation becomes the dominant excitation mechanism of the giant resonances for heavy ions above about $100 \mathrm{MeV} /$ nucleon. The Coulomb excitation provides extremely large differential cross sections for excitation of both isovector and isoscaiar resonances. Recent data are presented for inelastic scattering of 84 $\mathrm{MeV} /$ nucleon ${ }^{2} \mathrm{O}$ ions on $2 \mathrm{az} \mathrm{Pb}$ that demonstrate the effectiveness of Coulomb excitation in providing extremely large cross sections and peak-to-continuum ratios for the isoscalar giant quadrupole resonance and the isovector giant dipole resonance.

Following the title of this conference, we will discuss what we have learned from the use of heavy ion probes to excite giant resonances and, more importantly, what we may learn in the future. To begin however, it is interesting to note that it was almost exactiy 15 years ago that inelastic proton and electron scattering ${ }^{1-3}$ showed the systematic excitation of the isoscalar giani quadrupole resonance (ISGQR) in a number of nuclei. As a reference point for my later discussion I show in figure 1 the data from that early $\left(p, p^{\prime}\right)$ experiment ${ }^{1}$ and it is easy to see from t. se data why many in the nuclosp physics community were slow to accept the suggestion that new giant resonances had indeed been found. We found rather small peaks sitting on top of a large nuclear continuum and used an analysis based on the DWBA theory of direct ireactions. A contrast to the early light-hadron data of figure 1 is shown by the recentiy obtained ${ }^{4}$ spectrum of figure 2 from inelastic scattering of 334 MeV protons from $20 \mathrm{~Pb}$. The data were taken using the HRS facility at LAMPF and have an energy resolution of about $70 \mathrm{kEV}$. From this figure we can easily argue that progress has certainly been made in optimizing the reaction to the excitation of these broad high-excitation energy resonances. As you will see $z$ later in my ciscussion, we are now able to solidly eclipse even this beautiful

sotiom picture of giant resonances.

*operated by Martin Marietta Energy Systems, Inc. under contract

DE-ACO5-840R21400 with the U.S. Department of Energy.

The submoned mumuscrot hes beon

unhored by a contractor of the U.S

Governtuent und contrect No. DE-

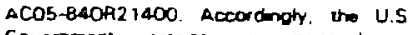

Govertment retent a nomexchuave.

royity-tice beonse to publath or reproduce

the publithed form of thes contribution, o Elow others 10 do so, for U.S. Goverrment 


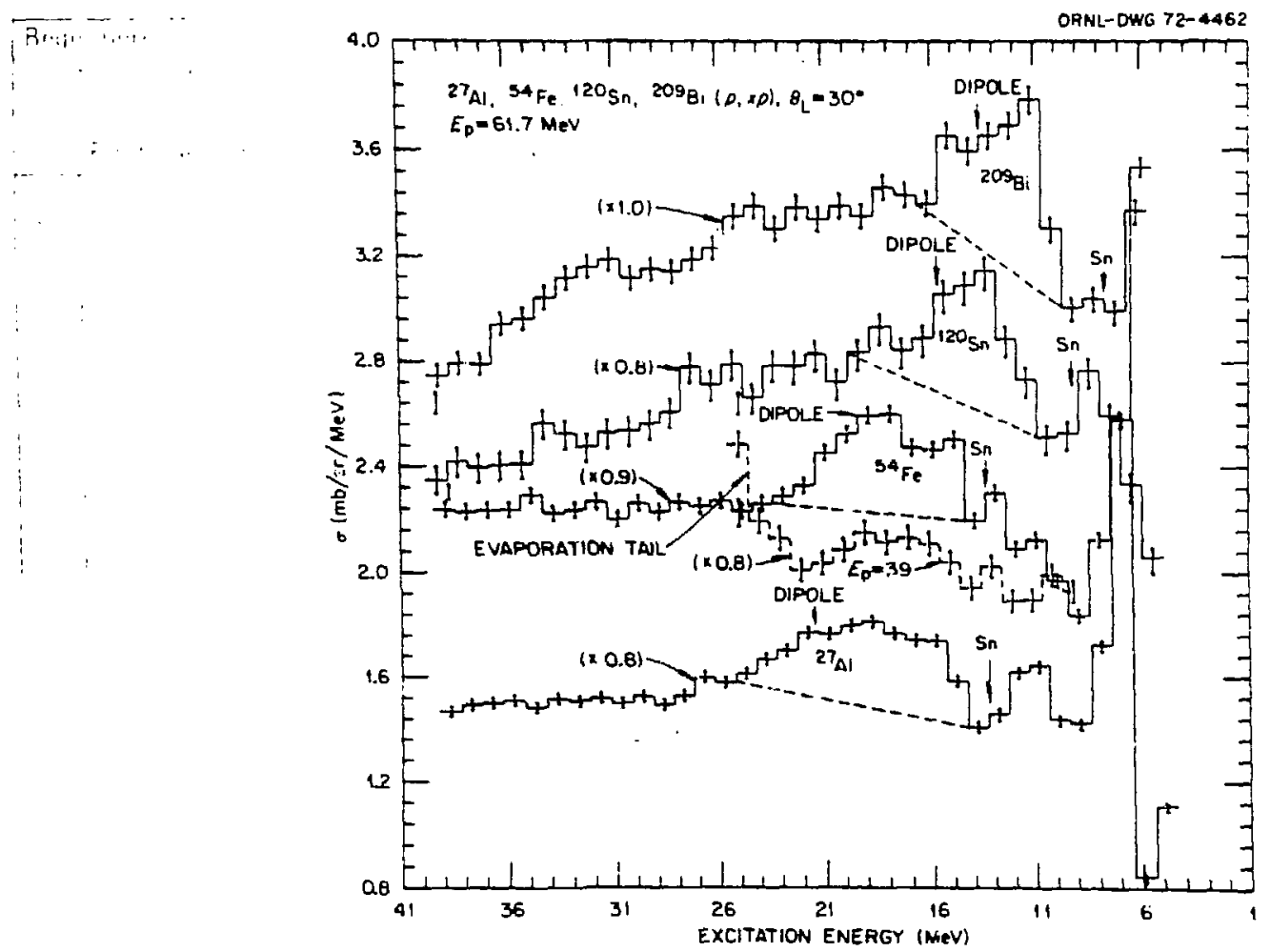

\section{FIGURE 1}

Inelastic proton continuum spectra at $30^{\circ}$ from ${ }^{27} \mathrm{Al}, 54 \mathrm{Fe},{ }^{120} \mathrm{Sn}$ and ${ }^{20} \mathrm{Bi}$ in the excitation region $\approx 6$ to $40 \mathrm{MeV}$. The spectra were taken at a bombardment energy of $E_{p}=62 \mathrm{MeV}$ except for the broken curve for ${ }^{5}$ "Fe which was taken at $E_{p}=39 \mathrm{MeV}$. The dashed 1 ines represent the approximation made for the underlying continuum in the region where an enhancement is observable. For convenience of plotting, the spectra include a scale factor as shown (Ref. 1).

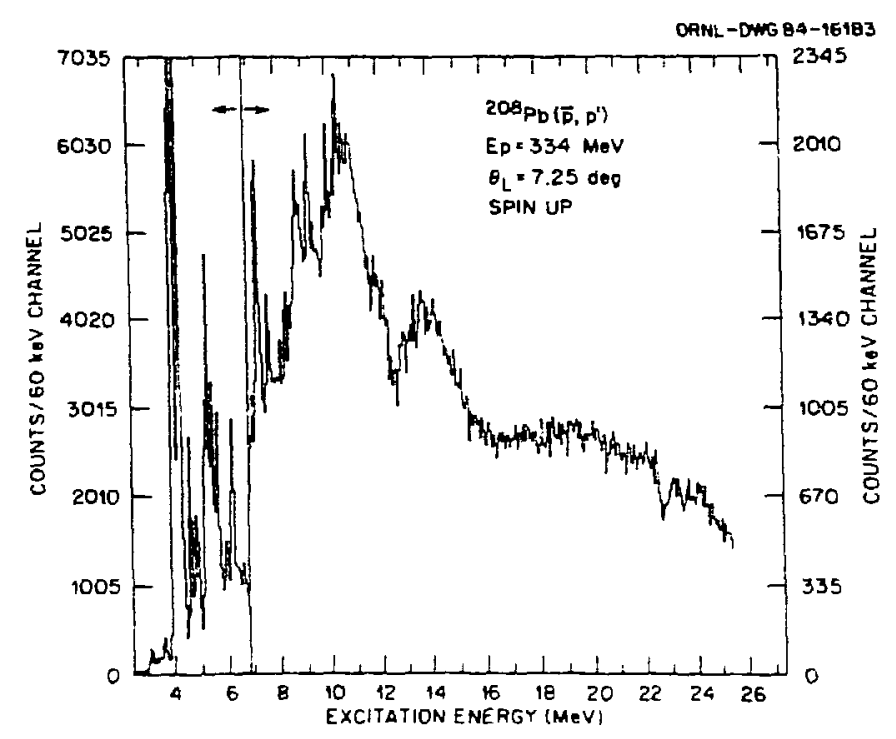

FIGURE 2

Inelastic scattering spectrum from $208 \mathrm{pb}$ at $7.25 \mathrm{deg}$. Note the scale change near 7 MeV (Ref. 4 ). 
Fine We imit our remarks to the use of inelastic scattering of hadrons to excite the giant resonances and will further consider only excitation of the electric giantrresonance modes, both isovector and isoscalar. With that in mind it is interesting to ask what resonances have been found in nuclei. Table 1 shows resonances in zospo which is surely the most heavily studied nucleus.

Table 1

Giant Resonances in $208 \mathrm{~Pb}$

\begin{tabular}{llll}
\hline Resonance & IS/IV & $\begin{array}{c}\text { Excitation } \\
\text { Energy }\end{array}$ & $\begin{array}{c}\text { Designation } \\
\text { in this Paper }\end{array}$ \\
\hline Dipole & IV & $13.6 \mathrm{MeV}$ & IVGOR \\
Dipole & IS & $17 ?$ & ISGDR \\
Quadrupole & IS & 10.6 & ISGQR \\
(Quadrupole) & IV & $22 ?$ & ISGQR \\
Monopole & IS & 13.9 & ISGMR \\
Monopole & IV & 32 & IVGMR \\
Octupole & IS & 20 & ISGOR \\
Hexadecapole & IS & 12 & ISGHR \\
\hline
\end{tabular}

While the evidence for ail these resonances is not uniformly strong, there is, nevertheless, at least reasonable evidence for them. As far as the IVGDR, ISGQR, and ISGMR resonances are concerned, data from virtually all hadron measurements are in agreement with one another and are in agreemont with the newest $\left(e, e^{\prime} n\right)$ measurements from Illinois. Most of the resonances listed in. Table 1 have been seen in a large number of nuclei and the systematic variation lof the energy of a given resonance with nuclear mass helps to provide evidence for the proper identification of a peak as a giant resonance.

While much has been learned about new giant resonances during the past 15 years there are still many open questions. For example, while there is good evidence for many isoscalar resonances, there is, except for the IVGDR, meager evidence for the existence of isovector, electric giant resonances. An exception to this statement is the IVGMR which has been found in a number of nuclei using the pion charge exchange reaction ${ }^{5}$. [Following the observation ${ }^{6-9}$ of the spin dipole resonance in $(p, n)$ charge-exchange studies, there have been recent reports ${ }^{10-12}$ of spin dipole strength in internediate energy ( $\left.p, p^{\prime}\right)$ studies.] There is very little data on high multipolarity $(L>3)$ resonances of isoscalar. wores isovector character. These resonances are likely to be very broad and, therefore, be difficult to observe experimentally. 
Fin How then does inelastic scattering of heavy ions fit into this picture? There have been severai ways suggested that heavy ions might enhance the study of giant resonances: $\cdots$ by providing larger cross sections, a larger peak-tocontinuum ratio due to a decrease of knock-out reactions, and the possibility to selectively excite resonances of high angular momentum. An example ${ }^{13}$ of some of the first studies of heavy ion excitation of giant resonances is shown on figure 3 and indicates that none of the hoped for gains were realized. These spectra are from $200 \mathrm{MeV}$. ( $17 \mathrm{MeV} /$ nucleon) $12 \mathrm{C}$ inelastic scattering from ${ }^{208} \mathrm{pb}$ and are shown along with a spectrum from $120 \mathrm{MeV}$ alpha particle inelastic scattering from the same target. In all the data a peak is observed at about $10.6 \mathrm{MeV}$, the energy of the ISGQR. The $-3 \mathrm{mb} / \mathrm{sr}$ cross section for the ISGQR in the ${ }^{12} \mathrm{C}$ spectrum is considerably less (- factor of ten) than that obtained for $120 \mathrm{MeV}$ alpha particle scattering. Furthermore, the peak-to-continuum ratio for the ISGQR in the carbon reaction is considerably poorer than that for the alpha particle spectrum. Clearly there is no enhancement of other, high multipolarity resonances in these heavy ion spectra. Overall these results could be considered disappointing.

More recently, accelerators have been able to provide heavy ions in the energy range of $20-30 \mathrm{MeV} /$ nucleon and experiments have been performed to excite giant resonances with those higher energy beams. It had been anticipated that the cross section for heavy-ion excitation of giant resonances should increase rapidly with increasing beam energy. In figure 4 we show spectra from the $\left({ }^{200},{ }^{10} 0^{\prime}\right)^{14}$ and $\left(a, \alpha^{\prime}\right)^{15}$ reactions on $208 \mathrm{~Pb}$ at $400 \mathrm{MeV}$ and $152 \mathrm{MeV}$ respectively. At $25 \mathrm{MeV} / \mathrm{nucleon}$ the giant resonance spectrum is much more prominent than it is at the lower heavy-ion energies. The cross section at the grazing angle is $\sim 40 \mathrm{mb} / \mathrm{sr}$, comparable to the $\left(\alpha, \alpha^{\prime}\right)$ cross section but the peak-tocontinuum ratio is much larger for the heavy-ion reaction than for the alpha particle reaction. However, note in the oxygen spectrum the large rise in cross section at excitation energies just below the of the quadrupole resonance. This large peak arises from excitation of states in the " 0 projectile and points out one of the serious problems with heavy-ion inelastic excitation of giant resonances; excitation of the projectile and nucleon pickup and subsequent rucleon decay of the projectile. The latter problem is shown in figure 5 where inelastic scattering spectra ${ }^{14}$ from 400 and $325 \mathrm{MeV} 160$ on $208 \mathrm{~Pb}$ are plotted. The location of the ISGQR is shown at $10.9 \mathrm{MeV}$ in both spectra. However, the prominent structures located between 20 and $40 \mathrm{MeV}$ clearly move with change in the incident energy of ${ }^{1} 0$ indicating that these peaks do not arise from excitation of states in $20 \mathrm{sb}$ but rather are caused by the projectile pickup and decay processes. It is possible to eliminate or at least 


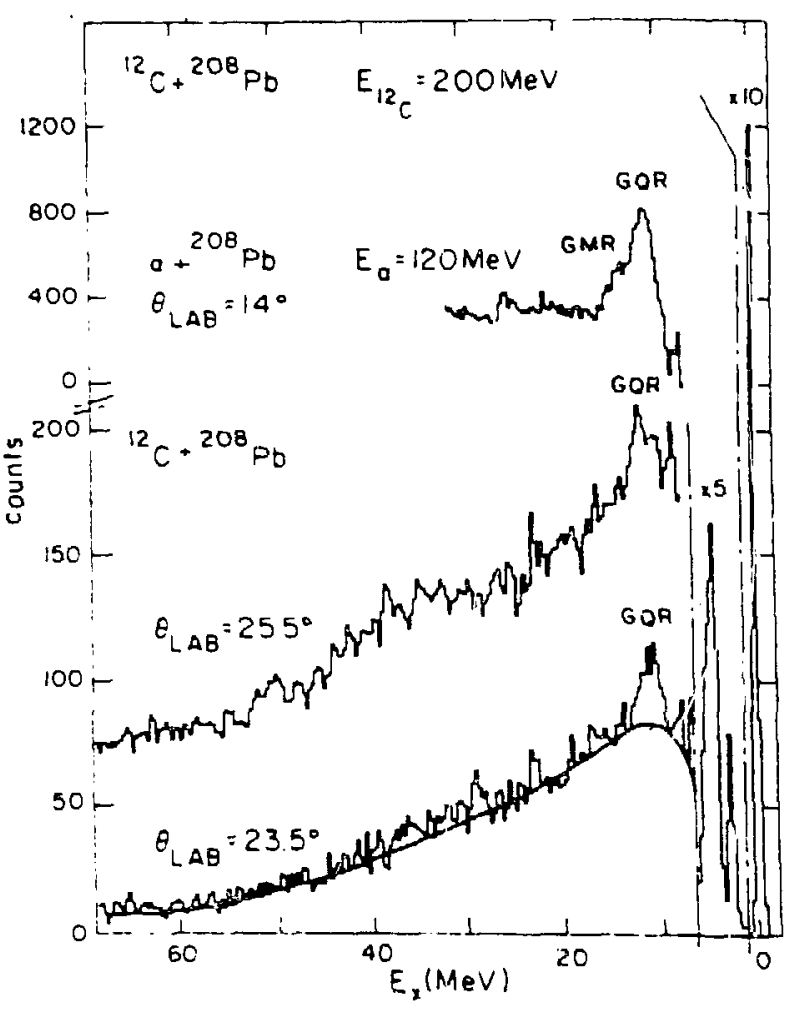

FIGURE 3

Spectra of the $208 \mathrm{~Pb}\left({ }^{12} \mathrm{C},{ }^{121}\right)^{208} \mathrm{~Pb}$ reactions at $E_{12} \mathrm{C}=200 \mathrm{MeV}$ and $208 \mathrm{~Pb}\left(\sigma, \sigma^{\prime}\right)^{203 \mathrm{~Pb}}$ at $E_{\alpha}=120 \mathrm{MeV}$ (Ref. 13).

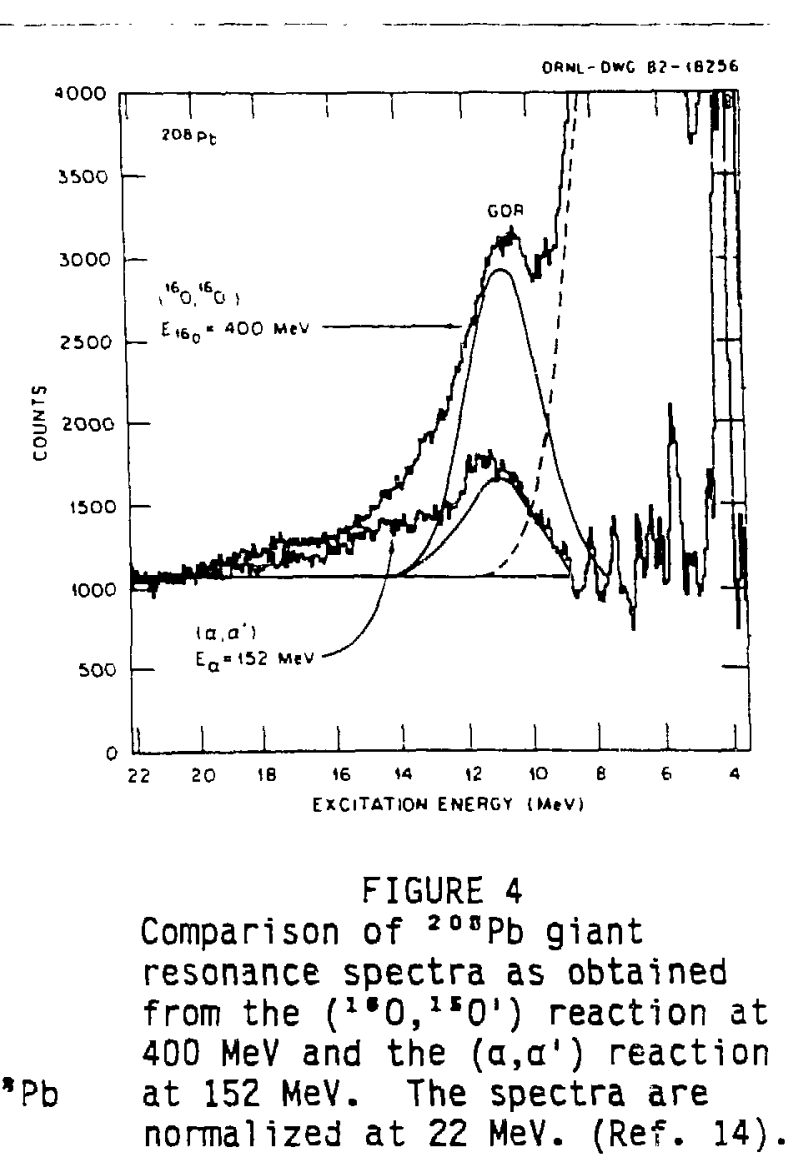

FIGURE 5

Inelastic spectra from the reaction $208 \mathrm{~Pb}\left({ }^{18} 0,{ }^{16} \mathrm{O}^{\prime}\right)$ at 400 - and $350-\mathrm{MeV}$.

The structure observed between $\sim 20$ and $\sim 45 \mathrm{MeV}$ of excitation shifts with projectile bombarding energy and is, thus, not from excitation of states in $208 \mathrm{~Pb}$ (Ref. 14). 
Substantial7y reduce contamination of the heavy ion inelastic spectra from piojestile excitation by choosing a projectile that is unbound to neutron emission at veriy low energy, projectiles such as ${ }^{13} \mathrm{C}$ or ${ }^{17} 0$. Figure 6 shows four inelastic scattering spectra 16 from $20 \mathrm{~Pb}$. Figure $6 \mathrm{~b}$ shows the same $\left(p, P^{\prime}\right)$ spectrum as in figure 2 while figure $6 c$ shows the 100 spectrum of figure 5. The spectrum in figure $6 a$ is from inelastic scattering of 376 MeV 170 and does not show the large peak at about. 6-8 MeV from projectile excitation as is the case for ${ }^{16} 0$ since ${ }^{17} 0$ is neutron unistable at about 4 MeV. The proton and 170 spectra are almost identical except for the region of the IVGDR. Finally, figure $6 d$ shows the inelastic spectrum from $22 \mathrm{MeV} /$ nucleon ${ }^{32} \mathrm{~S}$. Now one sees considerable structure in the giant resonance region that apparently arises from projectile not target effects. These spectra clearly demonstrate that care must be exercised in the choice of the heavy ion projectile used, and the energy of the heavy ion should also be considered to ensure that the excitation energy region of interest is not compromised by projectile eftects. These problems with heavy ion scattering are often difficult to detect and can lead to incorrect conclusions concerning the excitation of new states in nuclei.

While use of heavy ion inelastic scattering in the energy range of 20-30 MeV/nucleon clearly offers large cross sections and enhanced peak-to-continuum ratios, and profer choice of projectile can el iminate many of the effects of projectile excitation, it is still a fair assessmeni that all of the heavy-ion measurements to date show a selectivity similar to that observed with lighter probes. This is to say that the spectra are dominated by excitation of the ISGQR and the ISGMR. Furthermore, identification of the multipoiarity of the. giant resonance is difficult because the angular distributions for different angular momentum transfers in inelastic heavy-ion scattering are so similar.

Can we then expect any future for heavy-ion excitation of gianc resonances? We strongly suggest that the answer is yes. The answer lies in the use of still higher energy heayy ions where the effects of coulomb excitation become dominant over the nuclear interaction. Coulomb excitation by high-energy heavy ions will provide, as we shall see below, very large differential cross sections for inelastic excitation of the resonances. Furthermore, Coulomb excitation operates equally well on isoscalar and isovector states providing the possibility that for the first time, hadron scattering can provide strong excitation of isovector giant resonances.

z If we consider the coulomb excitation process in terms of the rapidly changing coulomb field created at the target by a projectile passing at high velocity in terms of the equivalent virtual photon field, then the coulomb 

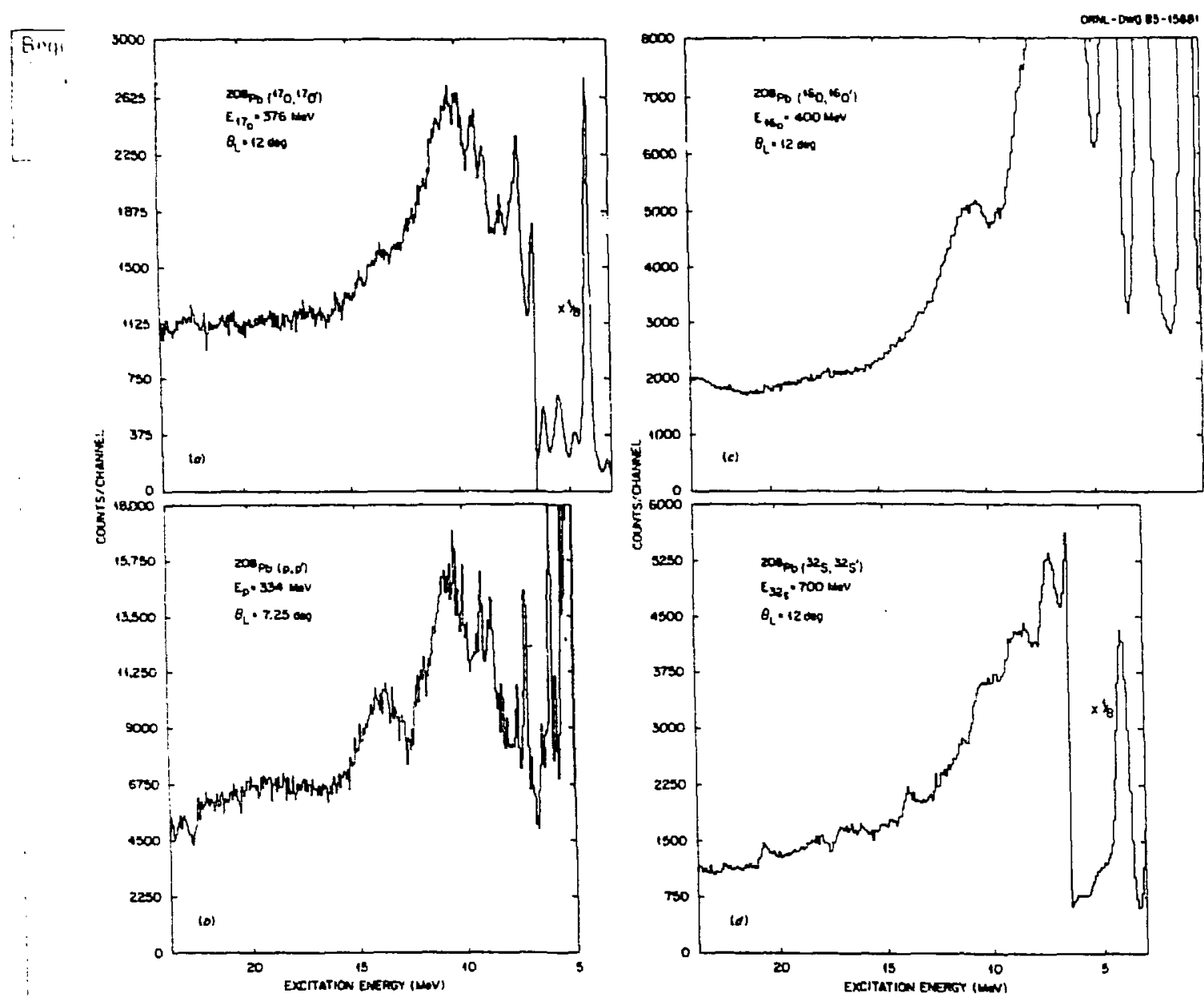

FIGURE 6

Inelastic scattering spectra for excitation energies between $\sim 3$ and $\sim 24 \mathrm{MeV}$. (a) $\left({ }^{17} 0,{ }^{7} 0^{\prime}\right), 12^{\circ}$, (b) $\left(p, p^{\prime}\right), 7.25$ (Ref. 8), (c) (160,160') $12^{\circ}$ (Ref. 6), and (d) (32s, 325'), $9^{\circ}$ (Ref. 16).

excitation process is equivalent to the absorption of the virtual photons by the nucleus. In figure 7 we show intensity spectra for electric dipole virtual photons as seen by a $20 \% \mathrm{pb}$ target for a range of 2.0 incident energies between 50 and $1500 \mathrm{MeV} /$ nucleon. At the lowest incident energies the virtual photon spectrum drops rapidly with increasing excitation energy and clearly shows why Coulomb excitation with low-energy projectiles is limited to the study of lowlying states. On the other hand, the spectrum at $1500 \mathrm{MeV} / \mathrm{nucleon}$ is almost flat in the excitation energy range of 10-50 MeV/nucieon. On figure 8 we compare the E1 virtual photon spectra from heavy ion reactions with that from 450 $\mathrm{MeV}$ electrons, long considered the cleanest probe as far as understanding the interaction is concerned. As one readily sees, the virtual photon spectrum :(per incident particle) in the 10-50 MeV range of excitation energy is comparably flat to that for the electrons and is more intense while offering the same understanding of the interaction since we are dealing with single step Coulomb excitation. 


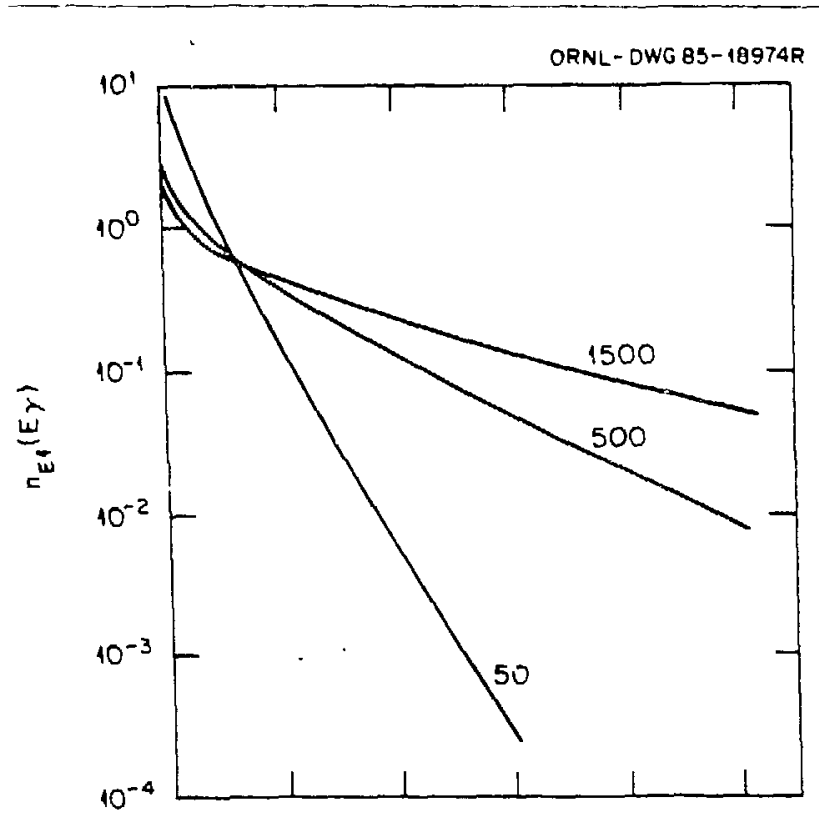

FIGURE 7

El virtual photon intensity spectrum seen by a $208 \mathrm{~Pb}$ target due to 50, 500, and $1500 \mathrm{MeV} /$ nucleon 10 0 beams.

ORML-DEC 97-11316

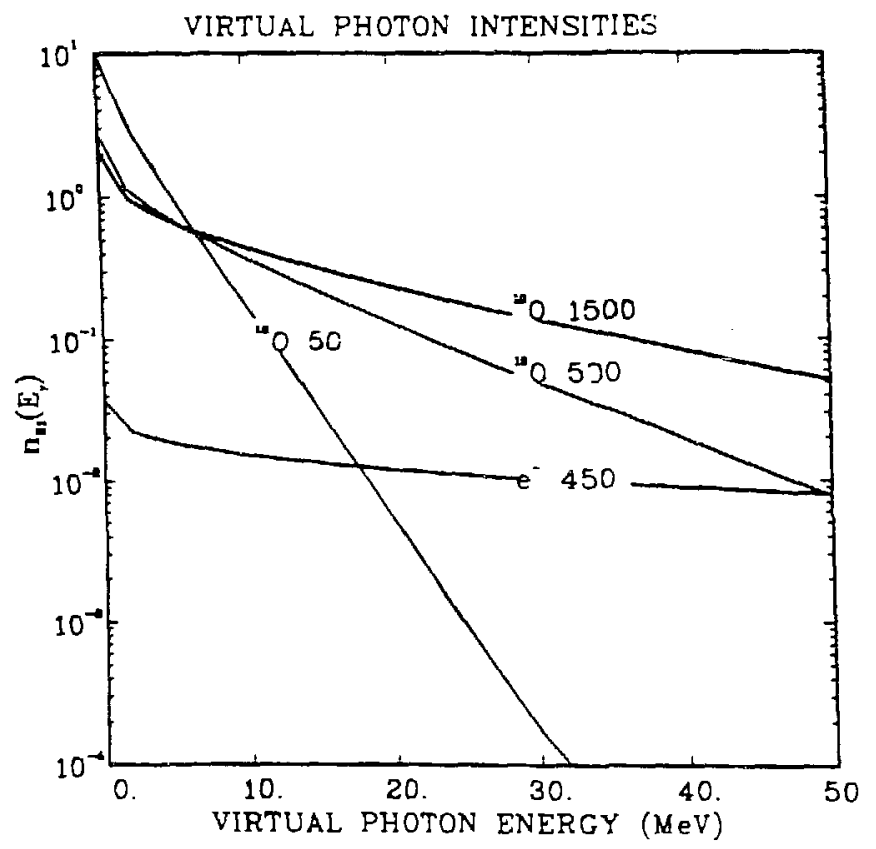

FIGURE 8

E1 virtuai photon intensity spectrum seen by a $208 \mathrm{~Pb}$ target due to 50, 500, and $1500 \mathrm{MeV} / \mathrm{nucleon}{ }^{2} \mathrm{O}$ jons and $450 \mathrm{MeV}$ electrons. 
Fopfhcoürse; the use of heavy ions is not 11 mited to 10 ns with $z \leq 8$ and since the Coulomb cross section increases as the square of the nuclear charge, one expects some, advantage in the use of heavier ions. Figure 9 shows the angle integrated cross section calculated assuming only coulomb excitation for excitation of the IVGDR in $20 \mathrm{PPb}$ by :hree different heavy ion beams as a function of the energy of the incident beam (MeV/nucleon). As one would expect, use of the heavy-ion beams increases the cross section for excitation of the state by orders of magnitude over the use of an oxygen beam. However, it should be noted that the problems associated with the use of heavy-ion beams, such as projectile excitation/decay, are still very much of concern at the higher energies and must be dealt with.

To make these calculations more relevant to experimental observation we show in figure 10 differential cross. sections for excitation of the ISGQR in $200 \mathrm{~Pb}$ for several incident energies of ${ }^{17} \mathrm{O}$. At the lower energies, 25 and 50 $\mathrm{MeV} / \mathrm{nucleon}$, the cross section is mainly from the nuclear interaction, while at the higher energies Coulomb excitation dominates. The effect of the increasing importance of Coulomb excitation with increasing incident energy is dramatic. The peak cross section for excitation of the ISGQR increases by over two orders of magnitude between $25 \mathrm{MeV} / \mathrm{nucleon}$ and $500 \mathrm{MeV} / \mathrm{nucleon}$, and would continue to jncrease at even higher energies.

Perhaps even more dramatic is the effect of the coulomb excitation on the excitation of the IVGQR as shown in the calculated differential cross sections on figure 11. The nuclear excitation of the isovector states proceeds through the isospin part of the nucleon-nucleus interaction which is quite weak in comparison with the strength of the non-spin and non-isospin term. On the other hand, the coulomb excitation is as strong for isovector states as it is for isoscalar states, all other things being equal. The cross sections shown in figure 11 are for excitation of an isovector state in $208 \mathrm{~Pb}$ at $22 \mathrm{MeV}$ by 170 at several beam energies. At $25 \mathrm{MeV} /$ nucleon the maximum cross section expectad for the IVGQR is about $1 \mathrm{mb} / \mathrm{sr}$, compared to a measured ${ }^{6}$ cross section of about $60 \mathrm{mb} / \mathrm{sr}$ for the ISGQR. On the other hand, at $500 \mathrm{MeV} /$ nucleon where the Coulomb excitation is dominant, the cross section for the IVGQR is calculated to be $10,000 \mathrm{mb} / \mathrm{sr}$ ! We point out in both figures 10 and 11 that the cross sections peak at smaller and smaller angles as the beam energy is increased (and the cross section increases) making experiments harder. These experiments will require the use of magnetic spectrographs and very clean, haloless beam. Figure 12 shows for $208 \mathrm{PD}$ the calculated ratio of the cross section for excitation of the ISGQR (10.6 MeV) to that for the IVGQR (22 MeV) as a function of the incident ${ }^{17} \mathrm{O}$ energy. For energies above $300 \mathrm{MeV} /$ nucleon the ratio of the cross sections approach one. When one considers that the cross section for 


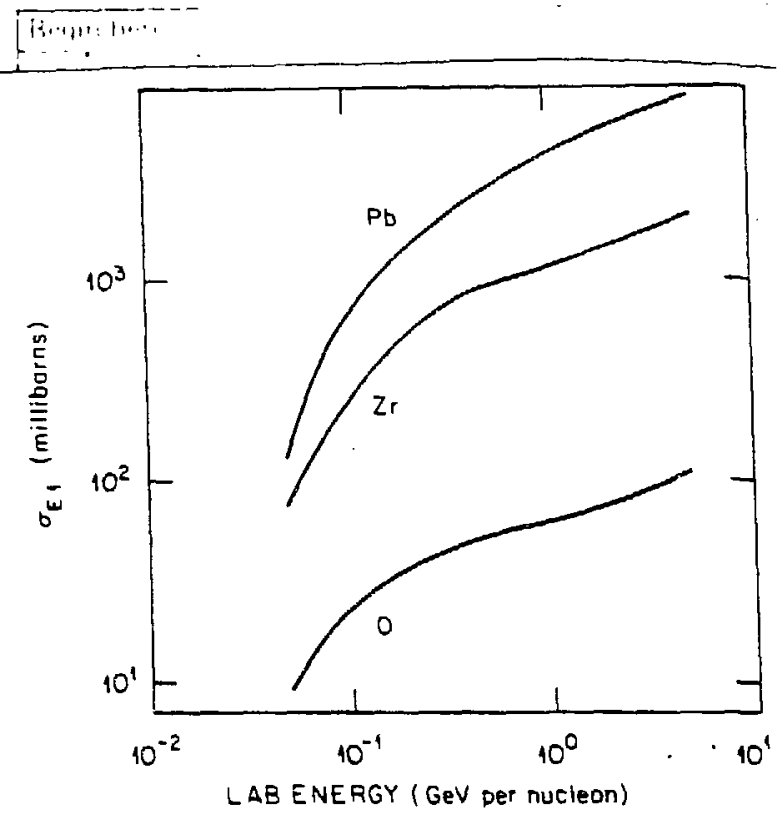

FIGURE 9

Cross sections for excitation of the IIVGDR state at $14 \mathrm{MeV}$ in ${ }^{208} \mathrm{~Pb}$ by various beams.

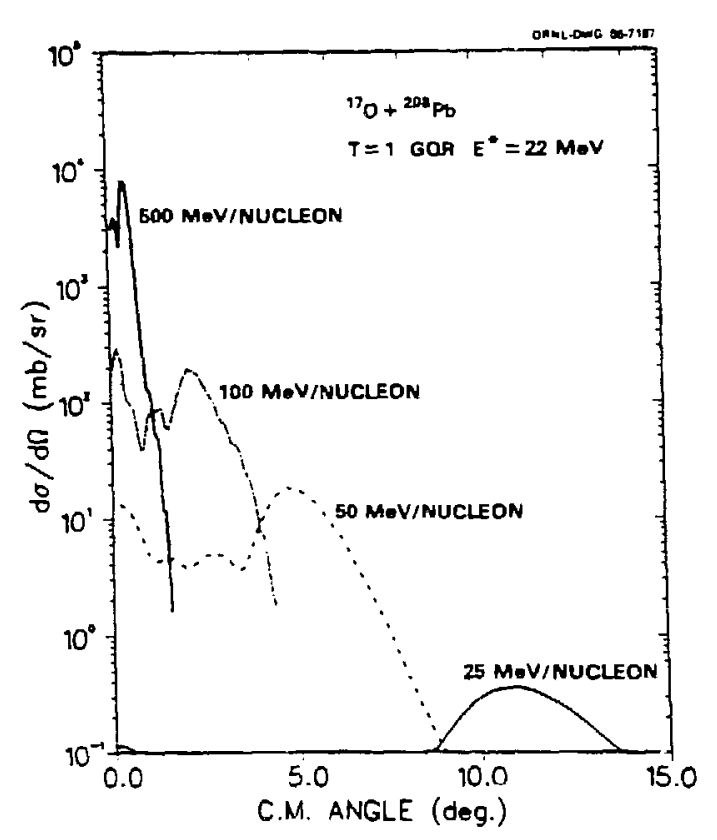

FIGURE 11

Calculated angular distributions for excitation of the isovertor giant quadrupole resonance in $208 \mathrm{~Pb}$ by inelastic scattering of 170 ions of several different energies.

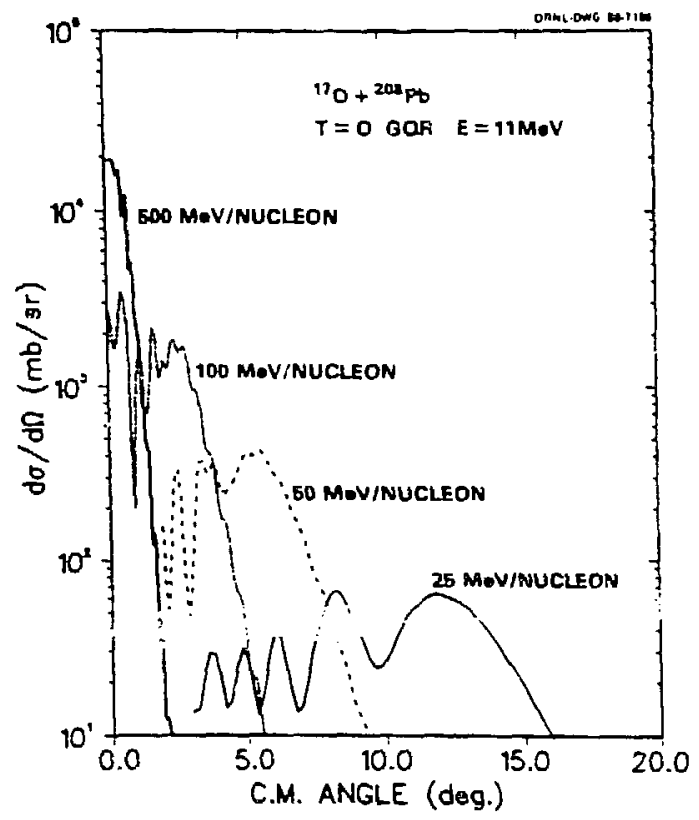

FIGURE 10

Calculated angular distributions for the isoscalar giant quadrupole resonance excited by inelastic scattering of various energy ${ }^{17} 0$ ions.

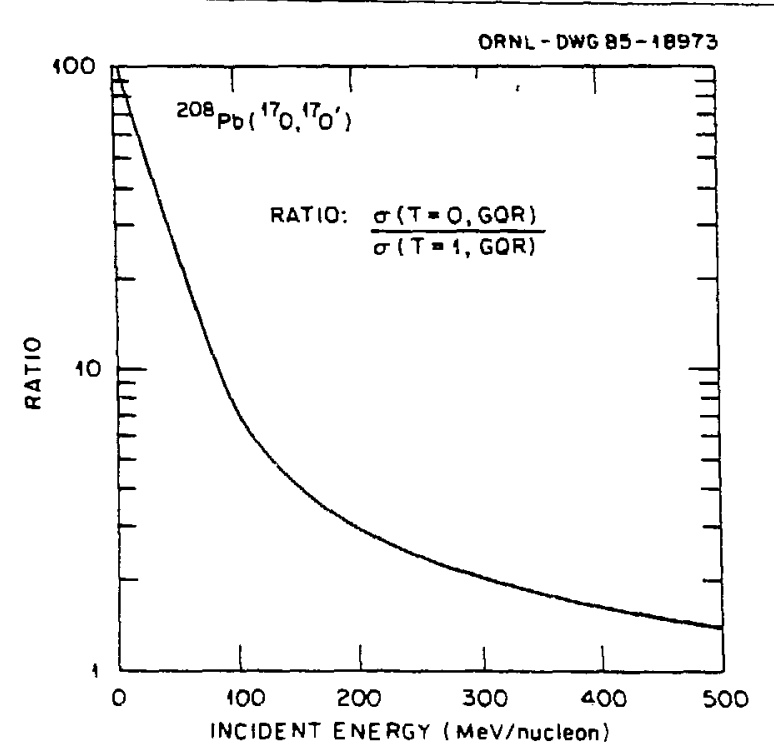

FIGURE 12

Ratio of calculated cross sections for excitation of the IVGQR (22 MeV) and ISGQR (10.6 MeV) by inelastic scattering of various energy ${ }^{17} 0$ ions. 
ThenISGQR is in the range of a few BARNS7SR at 500 MeVTnucleon, it is clear

that for the first time we may have a hadronic probe that provides large cross sectrions for isovector excitations. This may make possible studies of an entire class of giant resonances not yet available for systematic otservation.

Will we be able to carry out experiments using heavy-ion beams of the energies that seem to be needed to make what may be the next generation of giant resonance experiments? There are accelerator facilities presently under construction or proposed that can in principle carry out the measurerients suggested here. The SIS-18 accelerator at GSI is now under construction and will provide high quality heavy-ion beams at energies up to the range of a GeV/nucleon. The COSY accelerator at Julich is approved and could accelerate 1 light heavy ions to about a GeV/nucleon also. Proposals for acceleratcrs similar: to SIS-18 and COSY have been made by ORNL and Berkeley in the United States. Presently only GANIL has the beam eriergy and beam quality needed to begin to take advantage of the onset of the large coulomb excitation cross sections.

We have been involved in a research program at GANIL with collaborators from GANIL, Saclay, and Strasbourg in measurements of the excitation and nhoton decay of giant resonances excited by 84 MeV/nucleon ${ }^{17} 0$ beams. The phocon idecay measurements will be described in another contribution to this conference. We close this discussion with some of the results from the singles spectra obtained in those measurements.

Measurements $^{17}$ were made using the $84 \mathrm{MeV} /$ nucleon ${ }^{17} \mathrm{O}$ beam (full energy for (170) from the GANIL facility. Inelasically scattered 170 ions ware detected and identified in the energy loss magnetic spectrometer SPEG. The overall energy resolution was about $800 \mathrm{keV}$ due in large part to the use of a 5.1 $\mathrm{mg} / \mathrm{cm}^{2} 20 \mathrm{~Pb}$ targat. The spectrograph was set to accept events in the angular range from 1.5 degrees to 5.0 degrees. The momentum acceptance of the spectrometer is quite large, so that a single magnetic field setting provides data oyer an excitation energy range in excess of $70 \mathrm{MeV}$. Figure 13 shows a spectrum from the GANIL measurements (solid line) conpared to a spectrum, aiready shown in figure 6a, from $22 \mathrm{MeV} /$ nucleon measureinents at ORNL. The lower energy data is normalized to the GANIL data in the unstructured nuclear continuum (about $40 \mathrm{MeV}$ ). There are several obviously outstanding features in the GANIL data. First the giant resonance peak is huge, rising almost a factor of 10 above the continum. Reference to figure 6a shows that the 22 MeV/nucleon spectrin rises over a factor of two above the continuum as does the proton spectrum shown in figure 6b. Clearly, one of the objectives of the use: of heavy ions for giant resonance studies, the possibility of enhancing the peak-to-continuum ratio, has been realized. It is also apparent from figure 13 


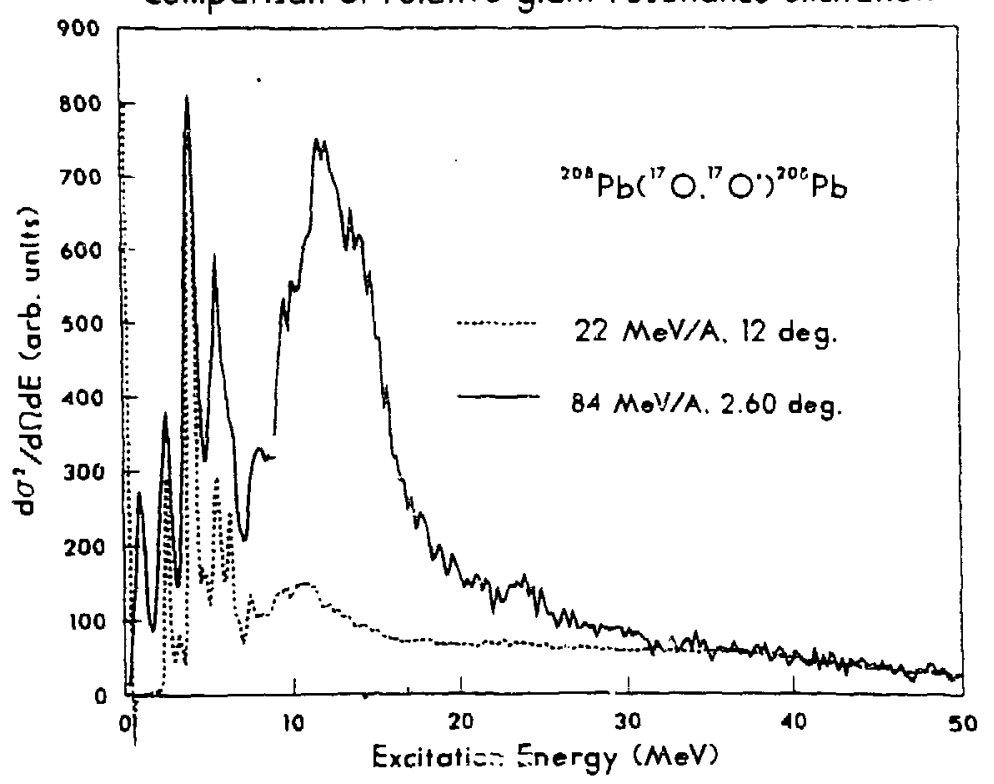

FIGURE 13

Spectrá from inelastic scattering of 84 and $22 \mathrm{MeV} / \mathrm{nucleon}{ }^{17} \mathrm{O}$ from ${ }^{208 \mathrm{~Pb}}$. The two spectra are normalized in the unstructured continuum near $40 \mathrm{MeV}$.

that the excitation energy of the centroid of the giant resonance peak in the GANIL data is higher than in the lower energy data. As will be shown below this is due to the fact that the coulomb excitation of the IVGDR dominates the ISGQR at the higher energy while the quadrupole resonance is the strongest excitation in the lower energy data. We note that the GANIL data i. wn in :figure 13 show the giant resonance peak and the peaks from excitation of $10 \mathrm{w}-$ lying states on the same arbitrary cross section scale. The peak height of the giant resonance structure is as large as that for the $4.08 \mathrm{MeV}, 2^{+}$level and larger than that for the $2.6 \mathrm{MeV}, 3^{-}$level.

The simplest question to ask concerning the composition of the giant resonance peak in the GANIL data is whether: we can account for the resonances previously observed to lie in the excitation energy region covered by the peak. We show on figure 14 an angular distribution for the entire peak ( $8 \mathrm{MeV}-16 \mathrm{MeV}$ ) shown in figure 13. We have assumed that the peak contains excitation of the IVGDR ( $100 \%$ EWSR), ISGQR ( $70 \%$ EWSR), ISGMR (100\% EWSR), and ISGHR (10\% EWSR). The solid curve on figure 14 is the sum of the cross sections for the excita-. tions we assume are contributing to the peak as calculated via the DWBA inclusing both coulomb and nuclear excitation. It is clear that we can account very well for the peak with our present knowledge of the location and strength of giant resonances from previous measurements. 


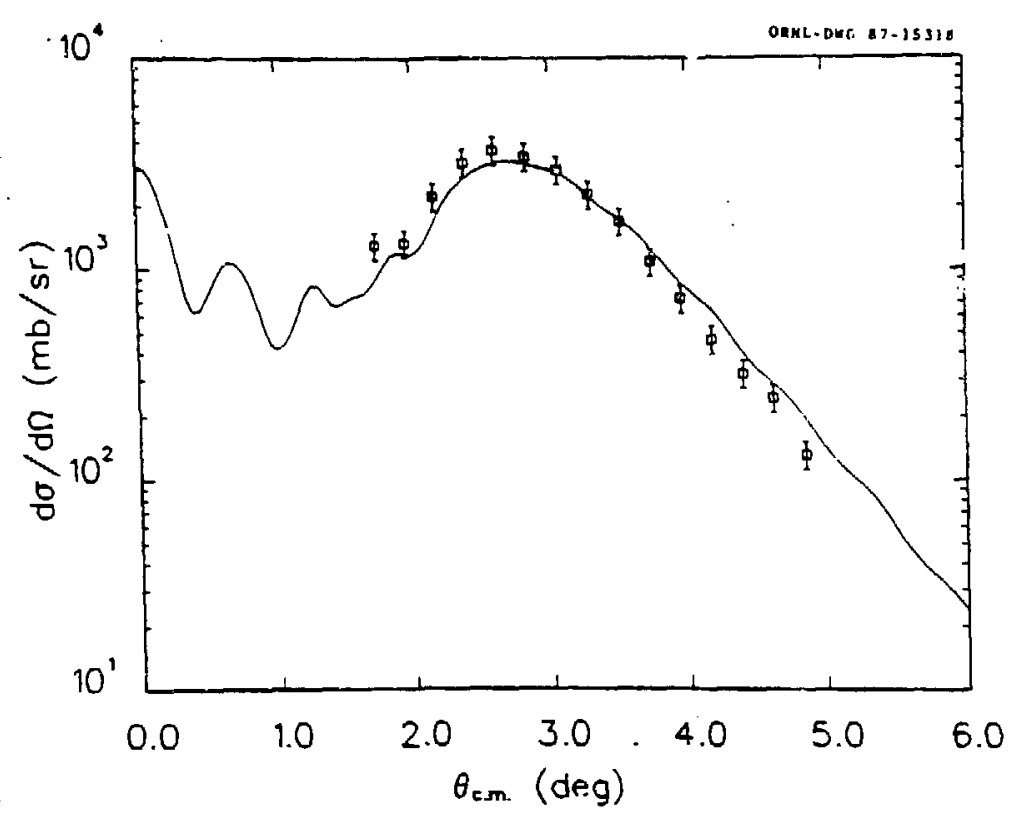

FIGURE 14

Angular distribution for "complete" giant resonance peak (fig. 13) integrated from 8-16 MeV. The solid curve is the sum of cross sections calculated using the DWBA for the ISGQR (70\% EWSR), IVGDR (100\% EWSR), ISGMR (100\% EWSR), and ISGHR (10\% EWSR).

The next step is to decompose the peak into the various components. However, before that is done it is important to discuss the effect of the Coulomb excitation on the shape of the giant resonance peaks. Reference to figure 7 shows that the E1 virtual photon spectrum is considerably flatter with excitation energy at beam energies above several hundred MeV/nucleon, than at lower energies of 50-100 MeV/nucleon where the Couiomb excitation probability drops very rapidly with increasing excitation energy. The drop is so fast that the excitation probability changes by over a factor of two across a peak as broad as the IVGDR. Thus, the shape of the IVGDR peak is considerably altered by the excitation mechanism from that observed in photonuclear work. Figure 15 snows the effect of the reaction mechanism on the shape cf the IVGDR for excitation by $84 \mathrm{MeV} / \mathrm{nucleon}$ (dotted line) and $22 \mathrm{MeV} /$ nucleon (dash-dot 1 ine) ${ }^{170}$ incident ions. The solid curve shows the shape of the IVGDR as taken from photonuclear measurements. As expected the effect is more dramatic at the lower energy. We have used the shape as shown in figure 15 in the analysis of the data from GANIL and from ORNL. The shape of the IVGDF as aitered ty the Coulomb interaction changes slightly with angle.

Figure 16 shows a spectra from the GANIL measurement; at three angles decomposed into peaks from the ISGQR, IVGDR, and ISGMR with centroids and widths taken from previous measurements and for the IVGDR, from the shape shown on figure 15. The areas of the peaks were allowed to vary and the shape of the underlying continuum was taken as generally flat under the peak. It is comforting to be able to say, for perhaps the first time, that, at least at most angles, the assumptions made about the shape and magnitude of the continuum underlying the giant resonances do not dominate the uncertainty in the data. 


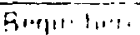

FIGURE 15

This figure shows the effect the rapidly changing Coulomb excitation probability as a function of excitation energy has upon the shape of the $20 \mathrm{~Pb}$ IVGDR. The solid curve is the dipole shape from photonuclear reactions. The dotted curve is the shape expected from excitation by $184 \mathrm{MeV} / \mathrm{nucleon}{ }^{170}$ ions and 'the dash-dotted curve is the shape expected for excitation by $22 \mathrm{MeV} /$ nucleon ${ }^{17} \mathrm{O}$ ions.

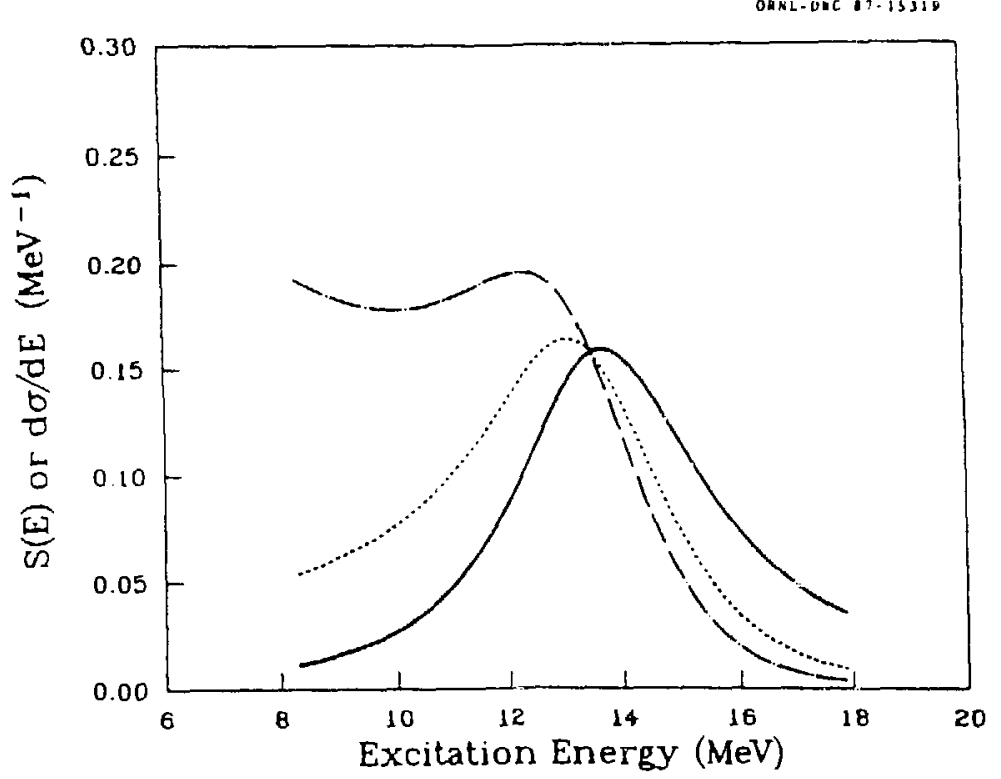

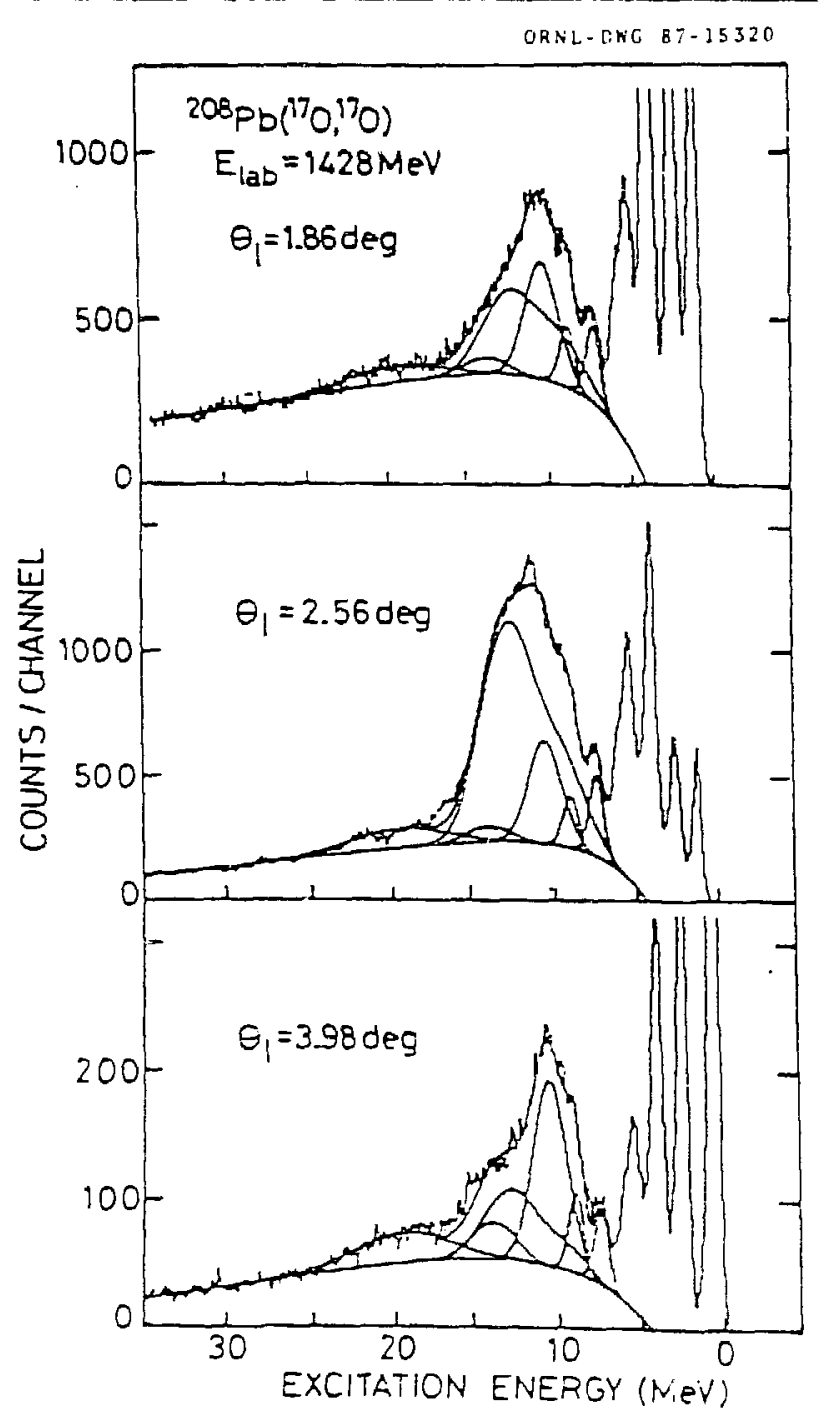

FIGURE 16

Inelastic scattering spectra at $\theta_{\text {ab }}=1.86^{\circ}, 2.56^{\circ}$, and $3.98^{\circ}$ from the $208 \mathrm{~Pb}\left({ }^{17} 0,{ }^{17} 0^{\prime}\right)$ reaction at $1428 \mathrm{MeV}$. The solid curves show a decomposition of the spectra into resonance peaks at $\sim 10.6 \mathrm{MeV}$ (ISGQR), $-13.6 \mathrm{MeV}$ (IVGDR), $14 \mathrm{MeV}$ (ISGMR), $7.5 \mathrm{MeV}, 9.1 \mathrm{MeV}$ and a broad, undefined, peak centered at $\sim 20 \mathrm{MeV}$ and an underlying continuum (Ref. 17). 
The "cross sections for the ISGMR and the ISGHR are quite sma7l relative to the cross section for the dipole and quadrupole resonances and for that reasen are quite uncertain and will not be dwelled upon in this discussion except to rote that the cross sections are consistent with previous measurements.

At most angles, excitation of the IVGDR dominates the spectrum as is seen in the decomposed spectrum in figure 16 . This is further pointed out by the angular distributions for the IVGDR and the ISGQR shown on figure 17 . The cross section for the IVGDR reaches a magnitude of nearly 3 barns/sr which is about four times the peak cross section of about $800 \mathrm{mb} / \mathrm{sr}$ for the ISGQR. These huge cross sections show that we have realized another hoped for attribute of heavy-ion excitation of giant resonances--very large cross sections. The calculation shown as the solid line on the dipole data was generated assuming only coulomb excitation of the IVGDR and the magnitude corresponds to $110 \%$ of the EWSR between 7 and $18.9 \mathrm{MeV}$. In the same energy range, ihe photonuclear reaction yields about $103 \%$ of the Eh'SR. We thus, account very well for the dipole excitation with the Coulomb interaction and resonance parameters from photonuclear data. The calculation for the ISGQR assumes both Coulomb and nuclear excitation as shown by the separate curves plotted on figure 16 . The angular distribution is well described by the $L=2$ calculation with a deformation length of 0.49 fm corresponding to exhaustion of $60 \%$ of the EWSR, in

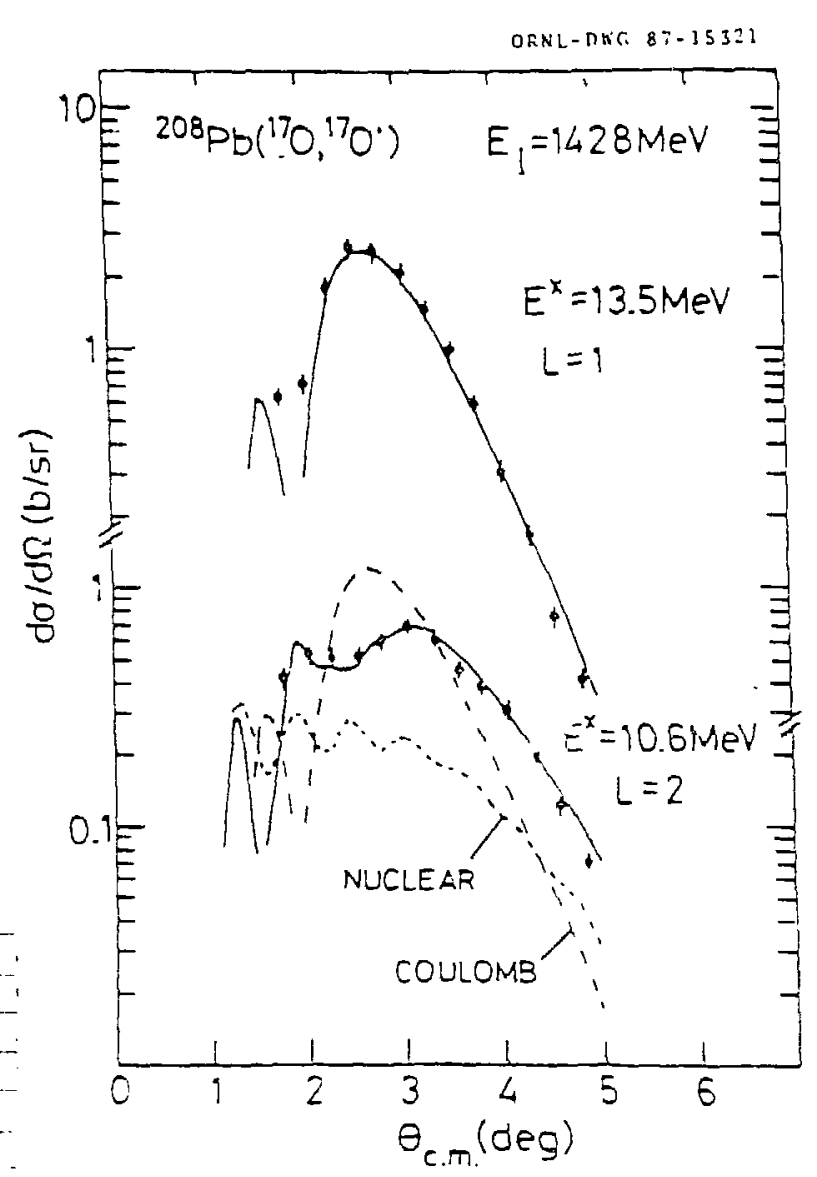

FIGURE 17

Experimental and calculated angular distributions for the isovector giant dipole resonance (upper part) and the isoscalar giant quadrupole. resonance (10wer part) in $20 \mathrm{Bp}$. For the ISGQR, the calculated coulomb and nuclear contributions to the cross section are shown (dashed lines) (Ref. 17). 
good agreement with previous data. The combination of excitation of the ISGQR via both Coulomb and nuclear interactions provides for a broader angula. distribution than is observed for the dipole where only Coulomb excitation is indicated. The observation of a large Coulomb excitation of the ISGQR is a strcng indication that its $B(E 2+)$ is close to the value expected for a state with $\sim 60 \%$ ISEWSR, and is inconsistent ${ }^{18}$ with the small B(E24) proposed ${ }^{19}$ for this resonance from $\pi^{+} / \pi^{-}$scattering.

The results from the GANIL measurements clearly show that our expectations for the lise of high energy heavy ions to excite giant resonances will indeed be met. Using an energy of only $84 \mathrm{MeV} / \mathrm{nucl}$ eon, the inelastic scattering of ${ }^{170}$ provides giant resonance cross sections and peak-to-continuum ratios that are far better than that achieved with any other probe to date. The fact that the IVGDR is so strongly excited clearly shows the utility of the higher energy heavy ions for excitation of isovector resonances. This suggests that the study of isovector resonances of higher multipolarity will be feasible when even higher energy heavy ions secome available. The huge cross section and peak-to-continuum ratio found for excitation of the dipole resonance make possible the study of the decay of the IVGDR (as we see in another presentation at this conference), an area of study that has heretofore beeri carried out only with inelastic photon scattering.

The use of high energy heavy ions for giant resoriance studies are not without drawbacks, such as an angular distribution insensitive to different angular momentum transfers, and the problems associated with the excitation of projectile and/or nucleon pickup and subsequent decay by the projectile. However, we believe that the heavy ion beams to become available from the new acceierators will provide new and exciting results for the next generation of giant resonance experiments.

\section{REFERENCË̈}

1) M. B. Lewis and F. E. Bertrand, Nucl. Phys. A196 (1972) 337.

2) R. Pitthan and Th. Walcher, Phys. Lett. B36 (1971) 563 .

3) S. Fukuda and Y. Torizuka, Phys. Rev. Lett. 29 (1972) 1109.

4) F. E. Bertrand, E. E. Gross, D. J. Horen, R. O. Sayer, T. P. Sjoreen, D. K. McDaniels, J. Lisantti, J. R. Tinsley, L. W. Swenson, J. B. MCClelland, T. A. Carey, K. Jones, and S. J. Seestrom-Morris, Phys. Rev. C 34 (1986) 45 .

J. D. Bowman, H. W. Baer, R. Bolton, M. D. Cooper, F. H. Cverna, N. S. P. King, M. Leitch, H. S. Matis, A. Erell, J. Alster, A. Doron, M. A. Moinester, E. Blackmore, and E. R. Sicilliano, Phys. Rev. Lett. 50 (1983) 1195. A. Erel1, J. Alster, J. Lichtenstadt, M. A. Moinester, J. 0. 
Bowman, M. D. Cooper, F. Irom, H. S. Matis, E. Piasetzky, U. Sennhauser, and $Q$. Ingram, Phys. Rev. Lett. 52 (1984) 2134.

6): D. E. Bainum, J. Rapaport, C. D. Goodman, D. J. Horen, C. C. Foster, M. B. Greenfield, and C. A. Goulding, Phys. Rev. Lett. 44 (1980) 1751.

7) D. J. Horen, C. D. Goodman, C. C. Foster, C. A. Goulding, M. B. Greenfield, J. Rapaport, E. Sugarbaker, T. G. Masterson, F. Petrovich, and W. G. Love, Phys. Lett. 95B (1980) :27.

8) D. J. Horen, C. D. Goodman, D. E.. Bainum, C. C. Foster C. Gaarde, C. A. Goulding, M. B. Greenfield, J. Rapaport, T. N. Taddeucci, E. Sugarbaker, T. Masterson, S. M. Austin, A. Galonsky, and W. Sterrenburg, Phys. Lett. 99B (1981) 383.

9) C. Gaarde, J. Rapaport, T. N. Taddeucci, C. D. Goodman, C. C. Foster, D. E. Bainum, C. A. Goulding, M. B. Greenfield, D. J. Horen, and E. Sugarbaker, Nucl. Phys. A369 (1981) 258.

10) C. Djalali, G. M. Crawley, B. A. Brown, V. Rotberg, G. Caskey, and A. Galonsky, Phys. Rev. C 35 (1987) 1201.

i1) C. Glasshausser, K. Jones, F. T. Baker, L. Bimbot, H. Esbensen, R. W. Fergerson, A. Green, S. Nanda, and R. D. Smith, Phys. Rev. Lett 58 (1987) 2404.

12) D. J. Horen, J. Lisantti, F. E. Bertrand et al., preprint (1987).

13) R. Kamermans, J. Van Driel, H. P. Morsch, J. Wilczynski, and A. van der Woude, Phys. Lett. B82 (1979) 221.

14) T. P. Sjoreen, F. E. Bertrand, R. L. Auble, E. E. Gross, D. J. Horen, D. Shapira, and D. B. Wright, Phys. Rev. C 29 (1984) 1370.

15) F. E. Bertrand, G. R. Satchler, D. J. Horen, J. R. Wu, A. D. Bacher, G. T. Emery, W. P. Jones, D. W. Miller, and A. van der Woude, Phys. Rev. C 22 (1980) 1832.

16) F. E. Bertrand, R. O. Sayer, R. L. Auble, M. Beckerman, J. L. Blankenship,

B. L. Burks, M. A. G. Fernandes, C. H. Glover, E. E. Gross, D. J. Horen,

J. Gomez del Campo, D. Shapira, and H. P. Morsch, Phys. Rev. C 35 (1987) 111.

17) J. Barrette, N. Alamanos, F. Auger, B. Fernandez, A. Gillibert, D. J. Horen, J. R. Beene, F. E. Bertrand, R. L. Auble, B. L. Burks, J. Gomez del Campo, M. L. Halbert, R. O. Sayer, W. Mittig, Y. Schutz, B. Haas, and J. P. Vivien, submitted for publication.

18) D. J. Horen, J. R. Beene, and F. E. Bertrand, to be published.

19) S. J. Seestrom-Morris, C. L. Morris, J. M. Moss, T. A. Carey, D. Drake, J. C. Dousse, L. C. Bland, and G. S. Adarns, Phys. Rev. 533 (1986) 1847. 